\title{
PHYSICAL PARAMETERS OF THE INTERMEDIATE AGE OPEN CLUSTER IC 1311: CLUES FOR THE THEORY OF STELLAR EVOLUTION
}

\author{
A. J. Delgado ${ }^{1}$ and E. J. Alfaro ${ }^{1}$ \\ Instituto de Astrofísica de Andalucía, CSIC Apartado 3004, E18080 Granada, Spain \\ Electronic mail: delgado@iaa.es, emilio@iaa.es \\ A. APARICIO \\ Instituto de Astrofísica de Canarias, Vía Láctea S/N E38200, La Laguna, Tenerife, Canary Islands, Spain \\ Electronic mail: aaj@iac.es \\ J. CABRERA-CAÑNO \\ Facultad de Física. Universidad de Sevilla Apartado 1045, E41080, Sevilla, Spain \\ Electronic mail: jesus@obelix.cica.es \\ Received 1994; May 18; revised 1994 August 2
}

\begin{abstract}
The results of a UBVR photometric study in the field of the open cluster IC 1311 are presented. A comparison with previously published results on NGC 7044, based on measurements secured during the same observing run, leads to self-consistent constraints to the parameters of the cluster. The location of selected samples of giant and unevolved dwarf members in the $U-B$ vs $B-V$ diagram is used to simultaneously compute the color excess $\mathrm{E}(B-V)$ and the metallicity $[\mathrm{Fe} / \mathrm{H}]$. Distance modulus and age are estimated by the ZAMS fitting method, and by the use of theoretical isochrones, based on models computed with and without the consideration of convective overshooting during the phases of core nuclear burning. The adopted set of values is: $\mathrm{E}(B-V)=0.28,[\mathrm{Fe} / \mathrm{H}]=0.0, \mathrm{DM}=13.9, \mathrm{Age}=1.6 \times 10^{9} \mathrm{yr}$. The mass function for the cluster main sequence has been estimated, with a slope $x=-2.58$, significantly steeper than the Salpeter IMF $(-1.35)$. The uncertainties in the adopted values for the cluster parameters, due to interstellar reddening, and to the calibrations of theoretical luminosity and effective temperature in terms of observational quantities, are also addressed. The discrepancies between the observational CM and the model isochrones, in particular the difference between observed and predicted luminosity of the RGC, are sensibly reduced for the models with convective core overshooting. The results indeed suggest the need of increasing the overshooting effectiveness beyond the values considered up to now in evolutionary computations.
\end{abstract}

\section{INTRODUCTION}

The significance of intermediate age and old open clusters as clue objects to the understanding of the formation history of the galaxy has been stressed many times in the literature. Their importance relies upon them being the representatives of the earliest events of star formation in the disk, and should therefore reflect, as a system, the physical properties of the galactic medium at the beginning of disk formation. In particular, the well tested interpretation of the differences in magnitude and color between main sequence turnoff and red giant branch (RGB) as age indicators allows reliable dating of the galactic disk, down to its earliest formation stages (see the recent report by Phelps et al. 1994). Furthermore, the precise determinations of distance, age and metallicity for these objects, provide one of the most useful tools to delineate, not only the global conditions, but also the local variations as well, in physical quantities of interest. We recall in this sense the results of Geisler et al. (1992), and Friel \&

\footnotetext{
${ }^{1}$ Visiting Astronomer, German-Spanish Astronomical Center, Calar Alto, operated by the Max Planck Institute fur Astronomie, Heidelberg, jointly with the Spanish Comisión Nacional de Astronomía.
}

Janes (1993), concerning significant local deviations from a galactocentric metallicity gradient, which might bear important implications for the processes involved in the formation and evolution of the galactic disk.

On the other hand, in the range of ages spanned by these clusters, the turnoff masses lie around the critical value, separating the regimes of helium flash and quiet onset of helium burning, a value which depends on the size of the convective core, and in turn, on the effectiveness, if any, of core overshooting during the phase of hydrogen burning. Stars counts in the red giant branch (RGB), red giant clump (RGC), and near the turnoff, should in principle provide constraints to this aspect of theoretical models (see the review by Chiosi et al. 1992).

However, the analysis of these issues collides with some bothersome facts, when real observations are to be compared with models to achieve quantitative estimates of the cluster parameters. On the theoretical side of the comparison between models and observations, the main differences between models arise from the opacities used, and, more important, with the not yet satisfactorily solved problem of a lacking physical theory of convection. But in addition, once theoretical tracks in the luminosity versus effective tempera- 
ture plane are obtained, the peculiarities of the calibrations used to transform them to the observational CM diagram, might cause non-negligible uncertainties in the comparison to observations.

On the other hand, in the reduction of observations we usually have to deal with interstellar reddening, which introduces at least two sources of uncertainty. First, due to varying reddening slopes and absorption coefficients with spectral type, in particular to the differences between dwarfs and giants, the discrepancy of the isochrone fits to $\mathrm{CM}$ diagrams cannot be uniquely interpreted in the sense of inaccuracy of the models. Second, photometric reddening and metallicity determinations are usually connected, which makes it inescapable that the choice of a particular reddening law affects the determination of metallicity. Obviously enough, the higher the reddening, the higher these uncertainties become. In particular, the poorly studied clusters, namely those located far outside the solar galactocentric radius, would be also those usually more affected by interstellar reddening, which makes its bothering effects to be larger, just when the insight intended with the observations should become deeper.

The problems in interpreting observations of highly reddened clusters has been illustrated in a recent paper by Aparicio et al. (1993, A93 in the following), on the cluster NGC 7044, whose results favor the inclusion of convectivecore overshooting in the evolutionary computations. In the present report, we deal with $U B V R$ CCD observations on the cluster IC 1311, performed in the same campaign, and reduced simultaneously with those in A93. This will provide some help in estimating the parameters of IC 1311, on the basis of comparisons between both clusters. The contents of the following sections are as follows: Section 2 contains a brief description of the observations and the reduction process. In Sec. 3, a first approach to the parameters of the cluster, based on the comparison with the results on NGC 7044 in A93, is performed, followed by the quantitative estimation of the relevant physical parameters: color excess, metallicity, distance modulus, age, and luminosity and mass functions for IC 1311. Section 4 includes a discussion of the results. We consider the possible influences of the above commented sources of uncertainty, and to what extent they might cause the observed discrepancies in the isochrone fitting to the dereddened magnitudes. Finally, Sec. 5 contains the main conclusions of our analysis.

\section{OBSERVATIONS AND DATA REDUCTION}

The cluster IC 1311 was included by Janes \& Adler (1982) in their list of rich, unstudied old open clusters, located in a direction far from the galactic center $\left(\alpha=20^{\mathrm{h}} 08^{\mathrm{m}} 48^{\mathrm{s}}, \quad \delta=41^{\circ} 04^{\prime}, \quad l=78: 4, \quad b=4^{\circ}: 2 ; \quad\right.$ Equinox 1950). The only existing photometric study of this object, based on $B V$ observations, was published by Alfaro et al. (1992). We shall comment later on the differences found between those results and the ones presented in this paper. The observations were performed in August 1989, using a 640 $\times 1024$ pixels, thin, uncoated, RCA CCD detector at the prime focus of the $3.5 \mathrm{~m}$ telescope at Calar Alto (Spain),

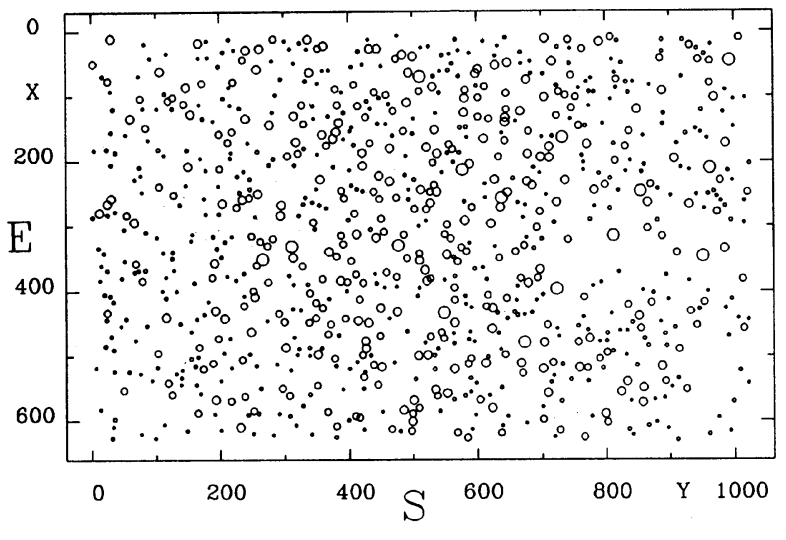

FIG. 1. Schematic representation of the star field covered in the observations.

giving a pixel angular size of 0.254 arcsec. Figure 1 shows a schematic representation of the field covered by the measurements, and Table 1 resumes the journal of observations. The results for 859 stars are listed in a table, available in the ApJ-AJ CDROM series. It includes an identification number, $X, Y$ coordinates, and at least one of $V,(U-B),(B-V)$, and $(V-R)$ (see Table 2 in A93).

As mentioned above, measurements in the field of the cluster NGC 7044 were secured in the same observing run. The process of reduction from raw data frames to standard magnitudes has been described in detail in the paper dealing with this cluster (A93), and will be briefly addressed here. IRAF, DAOPHOT, and ALLSTAR [see Stetson (1987) for the last two] were used to derive the magnitudes of the stars. A zero-point value was derived for each frame to transform these magnitudes into synthetic aperture ones (see A93). The errors affecting each zero point are 0.012 in $U, 0.008$ in $B$, 0.009 in $V$, and 0.005 in $R$. A set of 15 standard stars from the catalogs of Landolt (1983) and Neckel \& Chini (1980) were repeatedly measured in all nights, securing an adequate coverage in airmass, both for blue and red standards as well. Extinction reduction was performed by means of a so-called multinight method, based on the assumption that the magnitudes outside the atmosphere are constant for the individual standards, and computing every night not only the extinction coefficient, but also an extinction zero point, in each band, to met this requirement. Once the magnitudes outside the atmosphere are obtained, the following set of transformation equations was computed, and applied to the problem stars

$$
V=v+24.717-0.062(B-V) \text {, }
$$

TABLE 1. Journal of observations.

\begin{tabular}{ccccc}
\hline \hline Date & $\begin{array}{c}\text { Time } \\
(\mathrm{UT})\end{array}$ & Filter & $\begin{array}{c}\text { Exp. time } \\
(\mathrm{s})\end{array}$ & $\begin{array}{c}\text { FWHM } \\
\left({ }^{\prime}\right)\end{array}$ \\
\hline Aug.03.1989 & $23^{\mathrm{h}} 16^{\mathrm{m}}$ & $B$ & 1200 & 2.0 \\
Aug.03.1989 & $23^{\mathrm{h}} 41^{\mathrm{m}}$ & $V$ & 400 & 1.7 \\
Aug.03.1989 & $23^{\mathrm{h}} 52^{\mathrm{m}}$ & $R$ & 50 & 1.5 \\
Aug.04.1989 & $00^{\mathrm{h}} 27^{\mathrm{m}}$ & $U$ & 3600 & 2.1 \\
\hline \hline
\end{tabular}


TABLE 2. Errors in $B, V$, and $R$.

\begin{tabular}{cccc}
\hline \hline Mag & $\Sigma_{B}$ & $\Sigma_{V}$ & $\Sigma_{R}$ \\
\hline 16.0 & 0.00 & 0.00 & 0.00 \\
17.0 & 0.00 & 0.00 & 0.01 \\
18.0 & 0.00 & 0.01 & 0.02 \\
19.0 & 0.01 & 0.02 & 0.05 \\
20.0 & 0.02 & 0.04 & 0.14 \\
21.0 & 0.04 & 0.07 & $\cdots$ \\
22.0 & 0.08 & 0.15 & $\cdots$ \\
23.0 & 0.15 & $\cdots$ & $\cdots$ \\
\hline
\end{tabular}

$$
\begin{aligned}
& (U-B)=-2.779+1.094(u-b), \\
& (B-V)=-0.245+1.102(b-v), \\
& (V-R)=-0.156+1.241(v-r),
\end{aligned}
$$

where capital and small letters stand for Johnson-Cousins and instrumental magnitudes, respectively. The zero-point errors of the standard transformation can be found in A93. Adding them quadratically to those quoted above for the transformation of ALLSTAR to synthetic aperture magnitudes, the final zero-point errors of the photometry result 0.02 in $V$, 0.03 in $(U-B), 0.02$ in $(B-V)$, and 0.02 in $(V-R)$.

An analysis of the completeness and of the true errors as a function of magnitude has been performed for the $B, V$, and $R$ images, following the usual method of adding artificial stars to the observed frames, as described in A93. For each color, 480 artificial stars, distributed in 12 probes, have been analyzed. The results are summarized in Table 2 and in Fig. 2. Table 2 lists the errors $(\Sigma)$ affecting the photometry of the stars, in $B, V$, and $R$ (columns 2 to 4 ) as a function of the magnitude (column 1). Figure 2 shows the completeness level as a function of magnitude for each band.

\section{PHOTOMETRIC DIAGRAMS AND PHYSICAL PARAMETERS}

\subsection{Preliminary Evaluation: Comparison with NGC 7044}

In Figs. 3(a) to 3(c) we show the three CM diagrams of IC 1311 and NGC 7044. Both clusters were observed with the same equipment, in the same run, and the observations re-

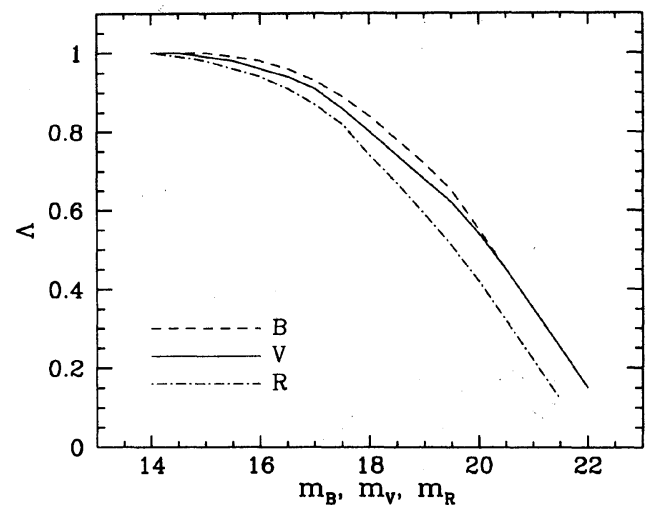

FIG. 2. Completeness curves for $B$ (broken line), $V$ (solid line) and $R$ (broken-dotted line). duced and transformed in the same way. Furthermore, the frames in both fields were taken at the same airmass, close to 1 , and with the same exposure times. This allows, in principle, to interpret the differences between CM diagrams, in particular those shown by the turnoff and giant stars, as being only caused by differences in the cluster parameters, and to achieve an internally consistent estimate for IC 1311. Three differences have to be mentioned here, however, namely, the obtaining of two frames for NGC 7044 in the $V$ band, with exposures of $400 \mathrm{~s}$ (the same as for IC 1311), and $800 \mathrm{~s}$ integration time, the larger angular size of IC 1311, 6', compared to $4.5^{\prime}$ for NGC 7044, as quoted by Janes \& Adler (1982), and the worse seeing conditions for the frames of IC 1311. Probably the last plays the most important role to account for the larger extent of the lower main sequence in NGC 7044.

Some significant features stand out in these plots: first, the different luminosity and color separations between turnoff and red giant clump (RGC), denoted in the following as $\Delta V_{\mathrm{to}, \mathrm{g}}, \Delta(U-B)_{\mathrm{to}, \mathrm{g}}, \Delta(B-V)_{\mathrm{to}, \mathrm{g}}$, and $\Delta(V-R)_{\mathrm{to}, \mathrm{g}}$; second, a bluer color of the unevolved main sequence, turnoff, and the RGC as well, for IC 1311; third, the main sequence in NGC 7044 appears to be wider in its upper part, close to the turnoff region, in particular in the $U-B$ index. In order to get a somewhat deeper insight from the comparison, we should rely as far as possible on member stars. For main sequence stars, we recall here the nonparametric membership discrimination method, devised by Cabrera-Caño \& Alfaro (1990), designed in principle to be applied to proper motions together with positions of stars in the field. The method is applied here to density distributions computed on the $\mathrm{CM}$ diagrams $V$ vs $U-B, V$ vs $B-V$, and $V$ vs $V-R$, by means of a bivariate Gaussian Kernel. A star is classified as a member when its membership probability is larger than $95 \%$ in all three CM diagrams [see Cabrera-Caño \& Alfaro (1990) for a detailed explanation]. This criterion is actually highly conservative, in the sense that it guarantees membership of the selected stars, but not all the rejected ones are nonmembers. As giant members, we select in each cluster those stars simultaneously belonging to the respective red giant clumps, in all three CM diagrams as well. The stars selected as members are plotted in Figs. 4(a)-4(c).

The distances in color and luminosity between turnoff and some representative point of the RGB are known to be mainly correlated with age, in the sense of both being smaller for younger clusters. The work published by Phelps et al. (1994) makes a fruitful use of these age indicators, and the recently published catalog of ages by Meynet et al. (1993, hereafter referred to as MEY) exemplifies the correlation quantitatively. Furthermore, the decrease of the color separation with decreasing age is stronger, the shorter the color index, as is clearly shown by the model isochrones of the Padova Group. (See for example, Fagotto et al. (1994), and references therein. We use here those isochrones already used by Carraro et al. (1993), kindly provided by the Padova group. We refer to them hereafter as PAD.)

All these features can be appreciated in the CM diagrams of Fig. 4, where we notice the different separations in magnitude between turnoff and the RGC, and in addition, that 

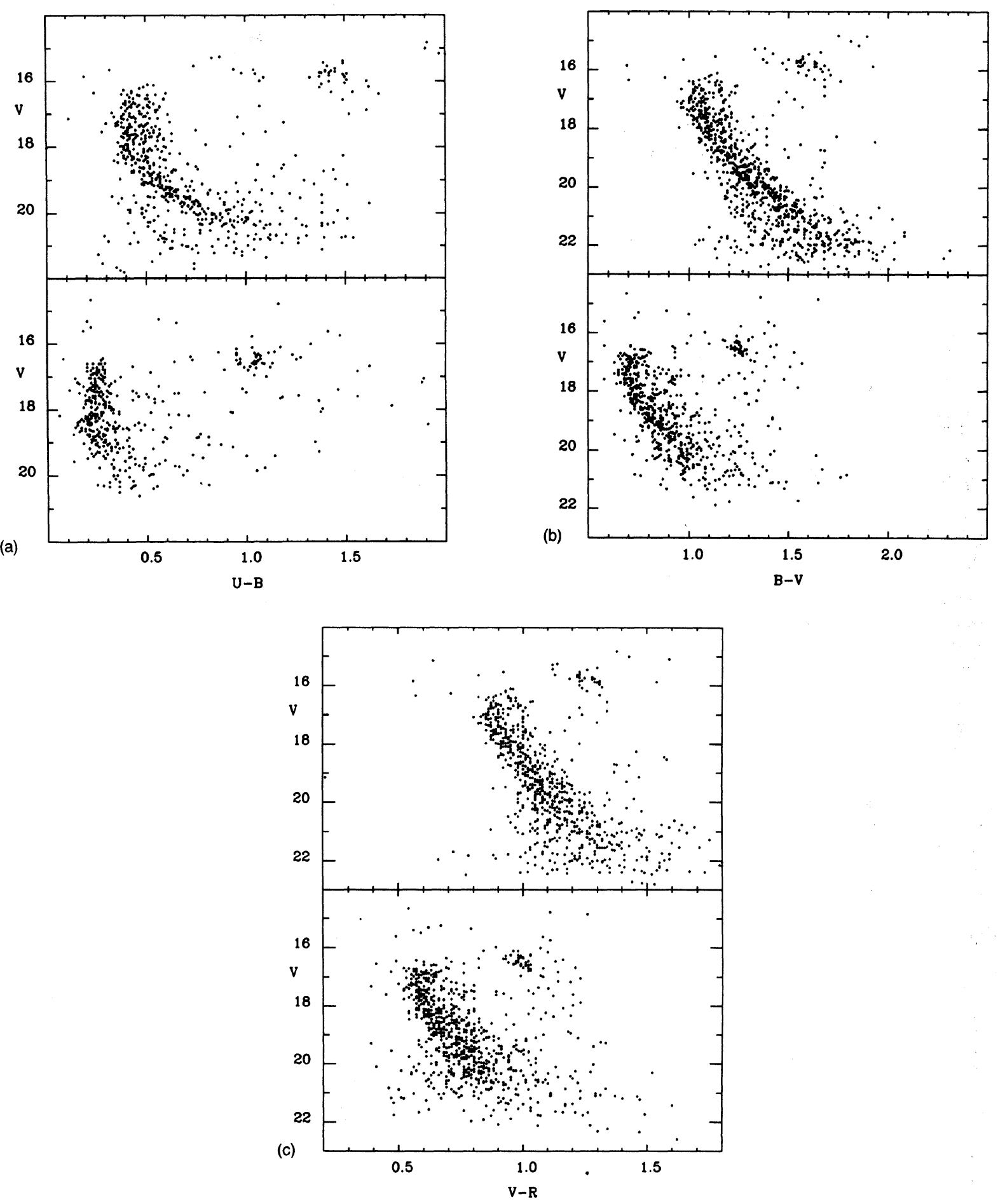

FIG. 3. Color-magnitude diagrams of IC 1311 in the $V$ vs $U-B, B-V$, and $V-R$ planes, compared to the same diagrams for NGC 7044 (Aparicio et al. 1993). The different separations in magnitude and color between turnoff and red giant clump can be seen.

$\Delta(U-B)_{\text {to,g }}$ is larger for IC 1311 than for NGC 7044, while $\Delta(B-V)_{\mathrm{to}, \mathrm{g}}$, and $\Delta(V-R)_{\mathrm{to}, \mathrm{g}}$ are similar for both. The combined evidences from the comparison of these morphological features in both clusters therefore indicate a clearly younger age for IC 1311 than for NGC 7044. The age value given for the latter by $\mathrm{A} 93\left(2.5 \times 10^{9} \mathrm{yr}\right)$ can be therefore considered as an upper limit. On the other hand, a run along the catalog by MEY shows the CM diagrams for NGC 2355 and NGC 2360 as the most similar to the one for IC 1311 presented here, which establishes the lower bound to the age of IC 1311 around $10^{9}$ yr.

We recall, however, that the specific age assignment in- 


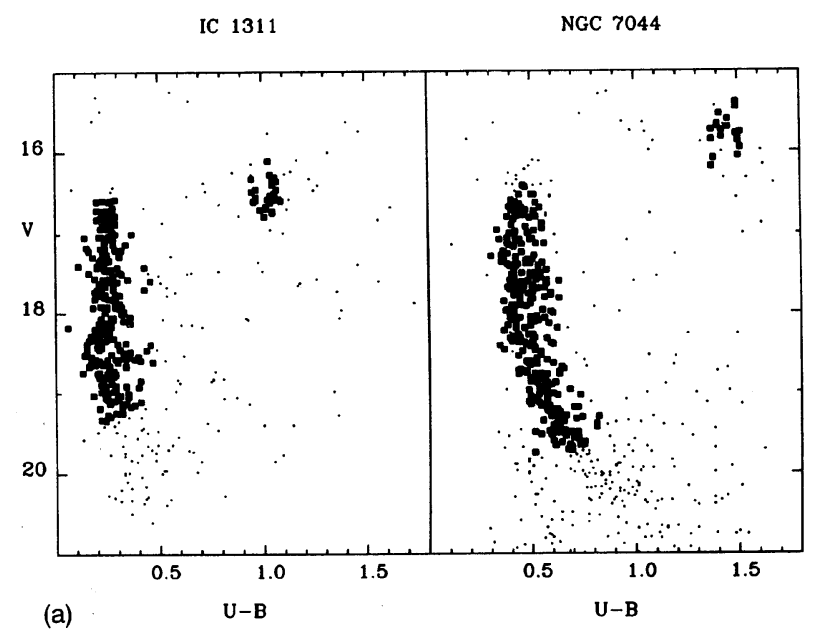

(a)

IC 1311

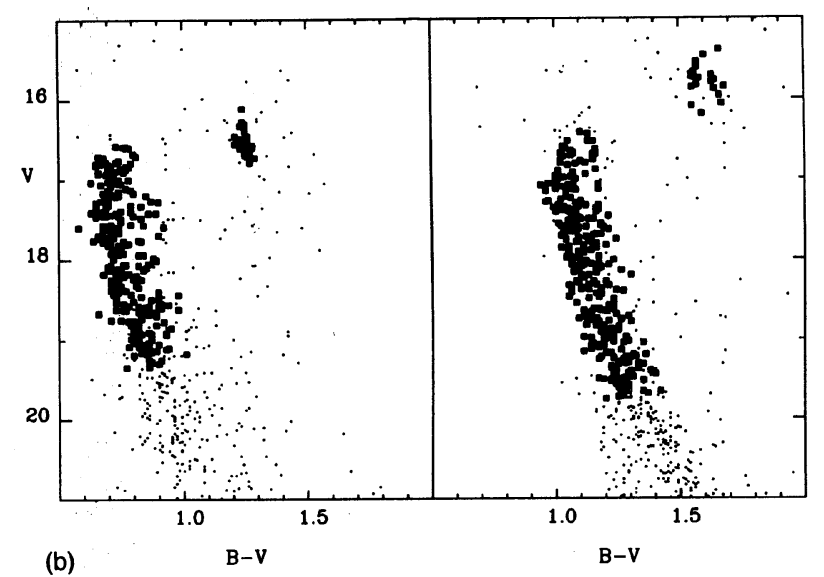

(b)

IC 1311

NGC 7044

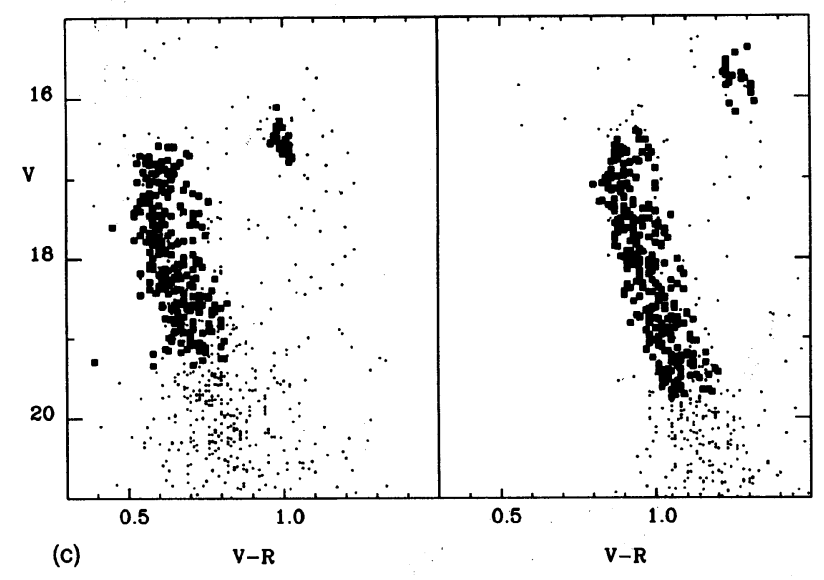

FIG. 4. Color-magnitude diagrams for IC 1311 and NGC 7044, with the member stars, selected through the membership discrimination method by Cabrera-Caño \& Alfaro (1990), plotted as black squares. The values plotted here are used to quantify the morphological differences between both clusters (see Sec. 3.1). deed depends on the set of model isochrones used, and that the comparison has to be carried out on the basis of intrinsic magnitudes and colors, if a quantitative estimate of age is to be achieved. This point is particularly relevant for highly reddened clusters, since the uncertainties in the reddening law could alter the conclusions reached on the basis of morphological comparisons between reddened CM diagrams.

As to the bluer CM diagram of IC 1311, it could be mainly a reflection of a lower value of the color excess, although a lower metallicity would act also in the same sense. On the basis of the values in the "members" plots of Fig. 4, the differences in $B-V$ and $U-B$ at the turnoff (in the sense NGC 7044-IC 1311) amount to 0.35 and 0.20 , respectively. The $U-B$ color of the brightest main sequence stars is known to vary much less than $B-V$ with age and/or metallicity changes. In fact, $\mathrm{PAD}$ model isochrones predict an inappreciable variation in $U-B$ of the upper evolved part of the main sequence, caused by these variations. Assuming the difference in $U-B$ in the upper main sequence to be a reflection of the color excess difference between both clusters, and a reddening slope $\alpha=\mathrm{E}(U-B) / \mathrm{E}(B-V)=0.72$, the difference in $B-V$ would be 0.28 , and the remaining 0.07 should reflect the younger age of IC 1311. The mixed effects of metallicity and age changes in the differences of turnoff colors, prevent further quantification, but the previous considerations suggest for IC 1311 a metallicity of the same order as the one of NGC 7044, and a color excess $\mathrm{E}(B-V)$ about 0.3 lower.

We finally comment on the apparent width differences between the evolved main sequences of both clusters, mainly seen in the $U-B$ color. This broadening could be interpreted as indicative of a higher binary content in NGC 7044 than in IC 1311. A closer look at Fig. 4, however, shows the red widening in the CM diagram of IC 1311 more sparsely populated and also more clearly detached from what can be seen as the high density main sequence locus. The fact that the width differences are more apparent in $U-B$ would be simply caused by the lesser varying $U-B$ color in the upper main sequence of IC 1311, resulting in the single and presumed binary representative points to join together. Assuming the widening of the evolved main sequence as due in part to binary stars in both clusters, the general aspect of the three $\mathrm{CM}$ diagrams would not suggest remarkable differences in the respective binary contents, but rather the possible presence of higher mass ratios for the presumed binaries in IC 1311 [see Aparicio et al. (1990) for a discussion on the effects of binaries in the CM diagram of star clusters]. On the other hand, considering the higher mean reddening affecting NGC 7044, a larger dispersion of color excess among individual stars in this cluster could also be expected.

Before turning to the direct computation of the photometric parameters for IC 1311, we just comment on the discrepancy between our first estimates here, and the results given in Alfaro et al. (1992). From their analysis, they conclude a much higher reddening, and a much lower metallicity value in turn $\{\mathrm{E}(B-V)=0.62,[\mathrm{Fe} / \mathrm{H}]=-0.4\}$. In addition to the lesser quality of the data used there, we recall here that these estimates were obtained without data in the $U$ band, and mainly on the basis of a direct morphological comparison 


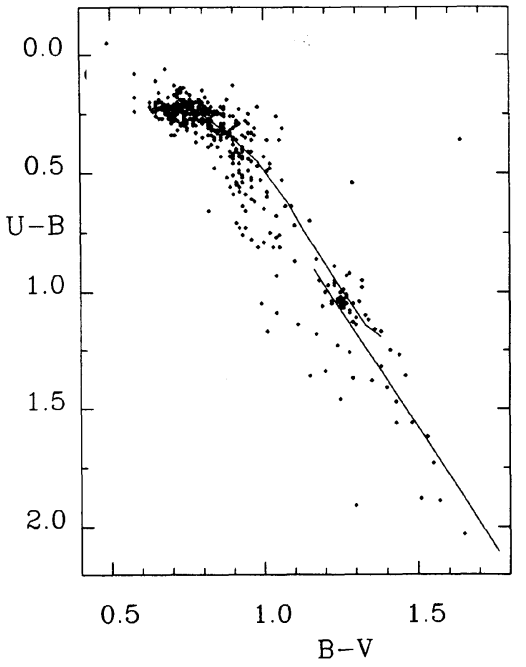

FIG. 5. Two-color diagram $U-B$ vs $B-V$ for the stars measured in the field of IC 1311. The Hyades reference lines for main sequence and giants are included, with a shift according to a color excess $\mathrm{E}(B-V)=0.28$.

with the CM diagram of NGC 2477 given by Hartwick \& Hesser (1974). The systematic differences between their color indices and ours amount to $0.073,0.132,-0.07$ in $V$, $B-V$, and $V-R$, respectively, which are to be compared with respective zero-point errors of $0.05,0.07$, and 0.08 . Their larger $B-V$ in particular, partly explains their result of higher reddening, but the largest discrepancy arises because of the lack of $U$ measurements, which made impossible the simultaneous estimate of reddening and metallicity from their photometry.

\subsection{Reddening, Metallicity, and Distance Modulus}

To carry out a quantitative estimate of the cluster parameters we apply the procedure devised by Twarog et al. (1993), already used in the analysis in A93. From the location of selected giant and nonevolved dwarf member samples in the two color $U-B$ vs $B-V$ diagram, color excess $\mathrm{E}(B-V)$ and metallicity $[\mathrm{Fe} / \mathrm{H}]$ are computed. The semiempirical ZAMS given by VandenBerg \& Poll (1989) is used to compute the distance modulus, through the ZAMS fitting procedure. The estimate of the cluster age is performed by comparing our dereddened $\mathrm{CM}$ diagrams with theoretical isochrones, whereby models with and without consideration of convective core overshooting are considered.

The computation of color excess and metallicity is based on the requirement that giant and unevolved dwarf cluster members have the same difference in $U-B$, so-called $\delta(U-B)$, with respect to the corresponding reference lines. The observational reference lines for giants and dwarfs in the Hyades are used (Sandage \& Eggen 1959; Boyle \& McClure 1975). Figure 5 shows the two color diagram for the observed stars. The Hyades ZAMS and giants reference lines are also plotted, shifted for a color excess $\mathrm{E}(B-V)=0.28$. As was done in A93, we use the location of stars in all three $\mathrm{CM}$ diagrams to select sets of giant and unevolved main sequence stars which can be considered as cluster members.

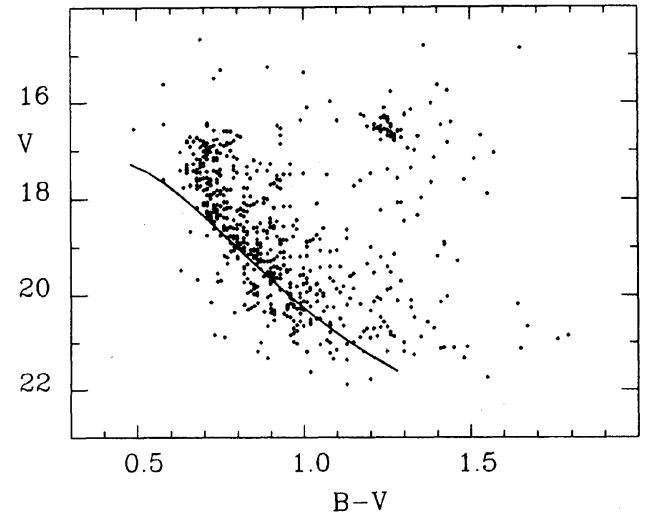

FIG. 6. ZAMS fitting to our reddened $V$ vs $B-V$ diagram. The ZAMS for solar metallicity by VandenBerg \& Poll (1989) are shifted to account for distance modulus and color excess $\mathrm{DM}=13.9, \mathrm{E}(B-V)=0.28$.

As giant members, all the stars belonging to the clump in the three diagrams simultaneously are selected. On the other hand, the selection of unevolved dwarfs is done by first considering the representative points inside a box 0.3 mag wide in $V$, above a lower envelope of the main sequence between $V=18$ and $V=20$, on the $V$ vs $B-V$ diagram. From these stars, only those also contained inside similar boxes in the $V$ vs $U-B$, and $V$ vs $V-R$ plots are retained.

$\delta(U-B)$ is computed by shifting the lines in the $U-B$ vs $B-V$ plane for different values of the color excess $\mathrm{E}(B-V)$, with reddening slopes $\mathrm{E}(U-B) / \mathrm{E}(B-V)=0.72$ and 0.9 for dwarfs and giants, respectively. The color excess for giants is thereby reduced by a factor 0.94 with respect to the one of dwarfs. The separations in $U-B$ from the reference line for main sequence stars are computed at $(B-V)_{0}=0.6$, for every value of the color excess, applying the corrections from the guillotine factors given by Sandage (1969) when necessary. With the above sketched procedure, we arrive at the following solution for the color excess and metallicity of IC 1311: $\mathrm{E}(B-V)=0.28, \delta(U-B)=0.03$. With the linear metallicity calibration of $\delta(U-B)$ given by Carney (1979), a metallicity value is obtained $[\mathrm{Fe} / \mathrm{H}]=-0.01$. The quadratic calibration in this same paper gives, $[\mathrm{Fe} / \mathrm{H}]=0.01$, whereas the use of the one given by Cameron (1985), leads to a somewhat lower value, $[\mathrm{Fe} / \mathrm{H}]=-0.07$. A metallicity value $[\mathrm{Fe} / \mathrm{H}]=0.0$ is adopted here.

Once the reddening and metallicity are established, a first estimation of the distance modulus can be performed, by means of the ZAMS-fitting procedure. The semiempirical ZAMS line for solar metallicity, given by VandenBerg \& Poll (1989) is used, which reproduces very accurately the fully empirical relation of Sandage \& Eggen (1959). Figure 6 shows the ZAMS line shifted to account for a true distance modulus $\mathrm{DM}=13.9$, compared to our $\mathrm{CM} V$ vs $B-V$ diagram. The absorption coefficients given by Buser (1978) have been used to shift the reference ZAMS line, as they produce a better general fit to the observed slope of the unevolved main sequence, as was already noticed in A93.

The estimates of reddening, metallicity, distance modulus, and age in turn, depend on the concrete reddening law used 
to compute the intrinsic magnitudes and colors. In A93, it was shown how the presence of high reddening leads to appreciable differences in the estimates, depending on the reddening slopes and absorption coefficients used. We have carried out the estimation of photometric parameters for IC 1311 using the reddening slopes for dwarfs and giants given by Buser (1978). The formal solution for color excess and metallicity is only slightly different from the one obtained above, $\mathrm{E}(B-V)=0.26$, and $\delta(U-B)_{0.6}=0.05$, which yields $[\mathrm{Fe} / \mathrm{H}]=-0.12$ through the linear relation of Carney (1979). The use of the ZAMS for this metallicity in the estimation of the distance modulus gives a somewhat lower value than the one quoted before, $D M=13.7$.

The range of possible values for the photometric parameters of the cluster lies within the observational errors, although an appreciable uncertainty still remains, particularly affecting the value of the distance modulus. The variations in reddening and metallicity, arising from the use of different possible reddening slopes, are, however, greatly diminished in this case, due to the lower color excess affecting IC 1311, allowing a reliable determination of its physical properties. Inverting the comparison between NGC 7044 and IC 1311 in Sec. 3.1, it can be taken as supporting the results adopted for NGC 7044 in A93, where the differences in the estimates from various reddening laws were significantly more relevant.

To finish this subsection, we comment on the presence of differential reddening in the field of IC 1311. The cluster was suggested to be embedded in a weak nebulosity by King (1964), and additional hints in this sense could be guessed from an apparent bimodality in the upper part of the main sequence, close to the turnoff region, where some groups of stars seem to delineate sequences detached from the main trend (see Fig. 3). These kind of features, already noticed in the observations of Alfaro et al. (1992), could reflect, among other things, differences in the color excess, depending on the location of stars in the field. We have looked closely to this possibility, by carrying out the estimation of color excess and metallicity for different subgroups of stars, as was done for the whole observed field. Neither the subdivision in concentric annuli around the estimated cluster center, nor the one in fringes both in north-south and east-west directions reveal appreciable traces of reddening variations across the cluster field covered by our frames.

\subsection{The Age of IC 1311}

An estimate for the age can be obtained through fitting of model isochrones to the dereddened CM diagram. As in A93, we use the sets of models computed with and without the consideration of convective-core overshooting during the phases of core nuclear burning, namely those from PAD and Castellani et al. (1992, hereafter referred to as CAS).

The renormalization procedure explained by Twarog et al. (1993), and used in A93 (see formulas 6 to 9 in A93 for details), is also applied here, to homogenize different sets of models, accounting for differences between them in chemical composition. As we only consider isochrones of solar metallicity, the shifts are computed to account for the helium con-

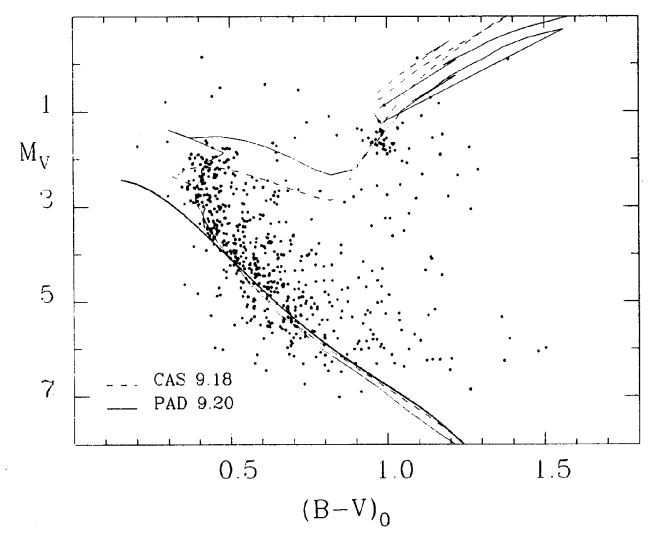

FIG. 7. Fitting of the PAD and CAS isochrones of the specified $\log A g e(\mathrm{yr})$ values, to the dereddened color-magnitude diagram $M_{V}$ vs $(B-V)_{0}$ of IC 1311. The absorption coefficients given by Buser (1978) have been used to deredden the stars.

tent differences, 0.28 in PAD, and 0.27 in CAS. On the other hand, the offsets applied by Twarog et al. (1993) and A93 to bring the predicted solar colors to the observed values are left aside here. Whereas the chemical composition is an input of the models, the differences in the predicted solar colors (or any other), are a result of the computation, i.e., obtaining of theoretical luminosity and temperature, plus transformation to the observational plane. In the next section we analyze more closely these differences between the PAD and CAS models, with the aim of elucidating to what extent they are reflecting the different treatment of the convective core.

In Fig. 7 we show the best achieved fit to the dereddened CM diagram with the isochrones of PAD and CAS, for $\log$ age $(\mathrm{yr})=9.2$ and 9.18, respectively, both for solar metallicity. The absorption coefficients of Buser (1978) have been used here to deredden the stars, with values of 0.28 and 13.9 for color excess and true distance modulus. We also plot here the main sequence from VandenBerg \& Poll (1989) for comparison. The global fit of the PAD isochrone is clearly better, in that both turnoff and location of the RGC are better reproduced. We notice in particular, that the differences in the agreement with the unevolved main sequence are well inside the errors inherent to the main sequence fitting procedure, whereas the difference in luminosity between the observed and predicted RGC positions is decreased when some amount of overshooting is included in the models. In the next section we look closer at some possible causes for this discrepancy.

\subsection{The Luminosity and Mass Functions}

Since for intermediate age and old star clusters, the approximation that all the stars were formed at the same time and under the same physical conditions can be assumed as valid, the analysis of their luminosity functions (LFs) and mass functions (MFs) should provide important constraints to the theories of star formation. In particular, the universality of the IMF, or whether it depends on physical conditions of the interstelar medium, like metallicity, density, position with respect to the galactic center and so on, could be tested 


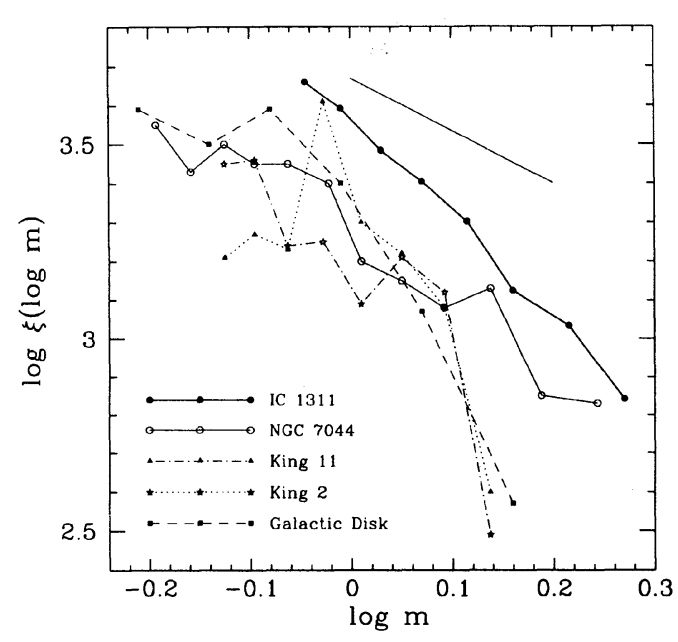

FIG. 8. The mass function of the stars of IC 1311, after correction for completeness, compared to those of NGC 7044, King 11, King 2, and the galactic disk. The straight line shows the Salpeter initial mass function with a slope $x=-1.35$. In the case of the clusters, $\xi$ refers to total number of observed stars. In the case of the galactic disk, the number of stars is normalized to the area projected on the galactic plane, in $\mathrm{pc}^{2}$. An arbitrary constant of 2 has also been added to the resulting logarithm.

through the analysis of the IMFs of the star clusters. However, an important limitation to this kind of analysis is the fact that the dynamical evolution of the clusters, producing mass segregation and dilution, modifies to some extent the initial conditions of these objects. Even though these mixed effects are difficult to disentangle, the systematic determination of LFs and MFs of clusters, and further comparative analysis, is certainly of interest.

Figure 8 shows the MF of the MS stars of IC 1311, after correction for completeness (see Fig. 2). We denote the MF as $\xi(\log m)$, which gives the logarithm of the number of stars normalized to the $\log m$ interval. Absolute magnitudes have been thereby converted into $\log m$, using data from Table IV of Scalo (1986). The mass functions of NGC 7044 (A93), King 11 (Aparicio et al. 1991), King 2 (Aparicio et al. 1990), the galactic disk (Scalo 1986), and the Salpeter IMF are also shown for comparison. A fit to the MF of IC 1311 results in a slope of $x=-2.58 \pm 0.09$, which is to be compared with the Salpeter canonical value, $x=-1.35 \pm 0.09$. We also recall here the value of $x=-1.66 \pm 0.06$ found by A93 for NGC 7044. From Fig. 8 it is apparent that, in the considered interval of the mass spectrum, the slopes of the $\mathrm{MF}$ of clusters and galactic disk are larger, in absolute value, than that of the Salpeter IMF.

\section{OVERSHOOTING VERSUS NONOVERSHOOTING MODELS}

It is by no means rare to find a discrepancy between the theoretically predicted position for the RGC, and the observed one. For instance, a look at the series of isochrones by MEY provides a good example. Their predictions of models for the RGC position are commonly off by typically 0.1 and 0.5 in color and luminosity, respectively. The disagreement seems to be larger the higher the color excess affecting the cluster, but the correlation is by no means clearly defined.
Although it always occurs in the CM diagrams of the clusters in the sample of MEY with higher reddening, some of the unreddened or slightly reddened clusters also show too faint RGC's as compared to the models.

We try now to elucidate which effects might be causing this disagreement, and to isolate the net influence of including core overshooting in the model computation. Different causes can be thought of to account for this discrepancy. First, we may recall the uncertainty in the reddening laws, already mentioned in Sec. 3.2, together with the errors in the photometry. To decrease the luminosity difference of the RGC with the models in our fits, we still could increase a few hundredths of a magnitude both distance modulus and color excess, to values of the order $\mathrm{DM}=14.0$ and $\mathrm{E}(B-V)$ $=0.3$, without contradicting with the distribution of representative points in the two color $U-B$ vs $B-V$ diagram, and keeping inside the errors quoted for the photometric indices. This, however, is not enough to eliminate the discrepancy of a too faint RGC as compared to theoretical predictions. On the other hand, to achieve full agreement between both we could also imagine an essentially larger absorption coefficient, $R=A_{V} / \mathrm{E}(B-V)$, for giants than for dwarfs. Considering that the difference, as estimated in Fig. 7, amounts to $0.31 \mathrm{mag}$ in $M_{V}$, and a color excess $\mathrm{E}(B-V)$ $\approx 0.3$, the value of $R$ should increase to at least 4.6, which does not seem justified. The values of $R$ obtained by Crawford \& Mandwewala (1976) for the Cygnus region, lie around 3.6 for giants, similar otherwise to those quoted by Buser (1978), in the spectral type range of interest here.

As a second possibility, the particular transformations used from theoretical effective temperature and bolometric magnitude, to the observational $M_{V}$ vs $(B-V)_{0}$ plane, might have some effect. The PAD models are transformed with theoretical colors and bolometric corrections (Carraro et al. 1993), whereas different combinations of empirical and theoretical calibrations by Flower (1977), Buser \& Kuruckz (1978), VandenBerg (1983), Malagnini et al. (1986), and Arribas \& Martínez-Roger (1988), are used by CAS and MEY in the range of temperatures covered by our turnoff and RGC. The net influence of the calibration upon the predicted morphology of the observational diagram can then be guessed by comparing the magnitudes and colors of PAD to those obtained by transforming their luminosities and effective temperatures with the particular combinations of calibrations used by these other authors.

Figure 9 shows the different results for the PAD isochrone of $\log$ age $(\mathrm{yr})=9.2$ (solid line). The dotted and dotted-broken lines represent this same isochrone, respectively transformed to $M_{V}$ and $(B-V)_{0}$, with the calibrations used by CAS and MEY. In addition, a shift to account for a helium content of 0.27 has been applied to all three lines (see Sec. 3.3). These three plots show acceptable agreement in the predicted location of the main sequence in the observational plane, whereas the color and magnitude predictions as well for the RGC may change by more than $0.1 \mathrm{mag}$. This variation in color is indeed important, and may lead to significant differences in the estimated cluster parameters by means of isochrone fitting, depending on which calibration from theoretical to observational quantities is considered. On the other hand, 


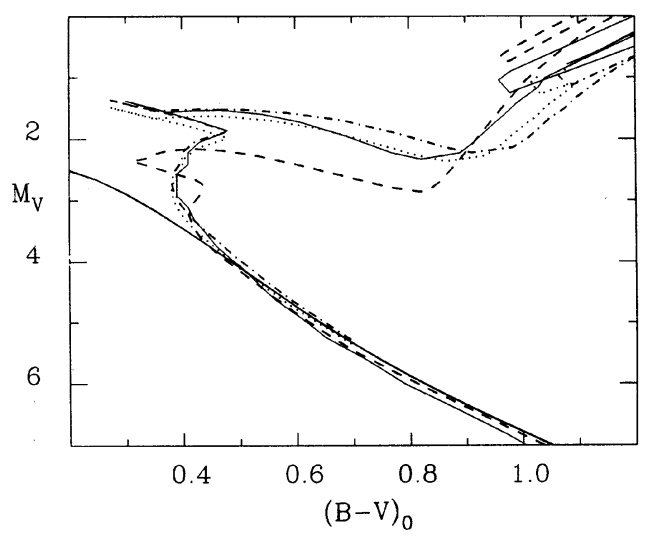

FIG. 9. Comparison of different calibrations of intrinsic magnitude and color in terms of theoretical luminosity and effective temperature. The isochrone of PAD for $\log A g e(y r)=9.2$, and solar metallicity (continuous line), is also transformed with the calibrations used by CAS and MEY (dotted and dotted-broken lines, respectively). The CAS isochrone for $\log A g e(y r)$ $=9.18$ (broken line), and the solar metallicity ZAMS from VandenBerg and Poll, are also shown.

a variation of $0.1 \mathrm{mag}$ in the predicted RGC absolute magnitude is still of the order of the observational errors. As far as can be tested, the differences between the currently used calibrations cannot therefore be argued as the cause for the discrepancy between theoretical and observed luminosity of the RGC.

In Fig. 9 we again include the CAS isochrone for $1.5 \mathrm{Gyr}$ as a broken line. The net effect of overshooting can be seen in the differences between the broken and the dotted lines. These two isochrones correspond to computations performed with the same chemical composition, the same opacities, and the same nuclear energy generation rates, and have been transformed to the observational plane with the same calibration. On the other hand, we can assume that other details of the input physics, such as equation of state and the technicalities of the computational codes, only produce negligible differences. The models represented by those two lines would therefore mainly differ in the prescription used to compute the size of the convective core. This comparison shows that overshooting is indeed able to decrease sensibly the discrepancy between predicted and observed luminosity of the RGC. In this sense, the previous considerations favor its inclusion in the evolutionary computations of stars in the pertinent mass range.

In addition to the better or worse quality of the global fitting of isochrones to the observed CM diagram, some further arguments on this point can be extracted from the comparison between theoretical and observed relative numbers of stars in different evolutionary states. The theoretical ratio of star numbers in any two given sections of the isochrones can be computed, assuming the initial mass function (IMF) $\xi(\log m)$ to be known. We assume here a power law form:

\section{$\xi(\log m) d \log m=a+x \log m d \log m$}

with $x=-2.6$, as estimated in Sec. 3.4. Since we only intend to derive relative numbers, the value of $x$ is not critical,
TABLE 3. MS/RGC predicted and observed rates.

\begin{tabular}{cc}
\hline \hline Model & MS/RGC \\
\hline CAS (1.5 Gyr) & 19 \\
CAS (1.0 Gyr) & 9 \\
CAS (0.8 Gyr) & 7 \\
PAD (1.6 Gyr) & 8 \\
PAD (1.3 Gyr) & 7 \\
PAD (1.0 Gyr) & 8 \\
IC 1311 & 5 \\
\hline \hline
\end{tabular}

but, in any case, the higher the $x$, the lower are the resulting ratios. Writing the mass function in a form dependent on $m$, instead of $\log m, \xi^{\prime}(m) d m=A m^{-3.6} \mathrm{dm}$, and integrating between the appropriate limits, the ratios predicted by the models are given by the equation

$$
R_{(\mathrm{MS} / \mathrm{RGC})}=\frac{m_{\mathrm{MS}^{1}}^{-2.6}-m_{\mathrm{MS}^{2}}^{-2.6}}{m_{\mathrm{RGC}^{1}}^{-2.6}-m_{\mathrm{RGC}^{2}}^{-2.6}},
$$

where $m_{\mathrm{MS}^{1}}, m_{\mathrm{MS}}$, and $m_{\mathrm{RGC}}{ }^{1}, m_{\mathrm{RGC}^{2}}$ denote the respective mass limits. Both for models and observations we consider the MS stars between 1 and 2 mag below turnoff, and the giants in an interval of $0.8 \mathrm{mag}$ above the base of the RGC.

The results for various CAS and PAD isochrones are compared in Table 3 to the observational ratio. Considering the error quoted for the slope of the IC 1311 mass function (see Sec. 3.4), an uncertainty of 0.8 can be estimated for the theoretical ratios. The observational ratio, $5 \pm 1$, is obtained from the set of member stars, selected in Sec. 3.1 (see Fig. 4), with completeness corrections according to Fig. 2. The relative error of about 0.2 can be estimated for the observational ratio as the inverse square root of the number of giants $(28$, compared to the MS number of 143). These results show a better agreement with the observed ratio for the prediction of the PAD isochrones. In particular, the difference is appreciable between the pair of CAS-PAD isochrones which best fit our dereddened CM diagram (Fig. 7), namely, those of 9.18 and 9.2 Gyr, respectively. The younger isochrones, both from PAD and CAS models, predict values for the ratio closer to the observational one, but they represent a too low age for the cluster. On the other hand, even considering the estimated errors, the observed ratio still remains less than the predictions. In this sense, we just recall here that the main sequence members selection, performed in Sec. 3.1, follows a very conservative criterion, and the observational ratio is therefore to be envisaged as a lower limit.

In absence of a reliable theoretical description of the phenomenon, the amount of overshooting and its detailed behavior with varying stars mass cannot be ascertained on physical grounds. However, on the basis of the considerations in this section, we see that the consideration of overshooting brings closer the predicted properties of the RGC to the ones observed in IC 1311, both in magnitude and in star number ratios. The results even suggest the adequacy of a slight increase of the overshooting efficiency, beyond the one in- 
cluded in the PAD models, at least for stars in the mass range covered by the upper main sequence and turnoff of the cluster.

\section{SUMMARY AND CONCLUSIONS}

The main results of our study can be summarized as follows:

(1) UBVR Johnson-Cousins photometry has been secured for 859 stars in the field of the intermediate age open cluster IC 1311. 643 of them have been measured at least in two bands. The analysis results in the following set of values for the parameters of the cluster: $\mathrm{E}(B-V)=0.28,[\mathrm{Fe} / \mathrm{H}]=0.0$, $\mathrm{DM}=13.9$, and Age $=1.6 \times 10^{9} \mathrm{yr}$. The variations of the cluster parameters, from the consideration of different reddening laws to obtain intrinsic colors and magnitudes, are of the same order as the observational errors. Furthermore, these adopted values also agree with the estimates deduced from a morphological comparison of reddened color magnitude diagrams of IC 1311 and NGC 7044 (Aparicio et al. 1993).

(2) A discrepancy is present between the model predictions and the observations, on the location of the red giant clump in the color magnitude diagram. Neither the uncertainties in the observations, due to observational errors and interstellar reddening, nor those arising from the differences between various possible $\left[M_{V},(B-V)_{0}\right]$ vs $\left(\log L, T_{\text {eff }}\right)$ calibrations, are able to completely account for it. This discrepancy is appreciably less pronounced for model isochrones based on evolutionary tracks computed with the consideration of convective-core overshooting.

(3) The rate of stars populating the red giant clump and a given section of the main sequence is used to test the convective-core overshooting vs the semiconvection formalism. The values obtained with models using the first one are in much better agreement with the rate observed in IC 1311. These conclusions jointly favor the use of the overshooting algorithm. Our results further suggest the possibility of a larger efficiency than the one included in the models, for stars in the mass range under consideration.

(4) The luminosity and mass functions of IC 1311 have been obtained. A power law fit to the MF results in a slope of $x=-2.58 \pm 0.09$. This value agrees with those found in other old and intermediate age open clusters, and in the galactic disk by Scalo (1986), in that they are significantly steeper than the Salpeter canonical IMF slope, $x=-1.35$.

This work has been financially supported by the Spanish DGICYT, through Grant number PB91-053, the Instituto de Astrofísica de Canarias, and the Research and Education Council of the Autonomous Government of Andalucía (Spain). The observations have been taken at the GermanSpanish Observatory of Calar Alto (Spain).

\section{REFERENCES}

Alfaro, E. J., Aparicio, A., Delgado, A., García-Pelayo, J. M., \& CabreraCaño, J. 1992, AJ, 103, 204

Aparicio, A., Alfaro, E. J., Delgado, A. J., Rodríguez-Ulloa, J. A., \& Cabrera-Caño, J. 1993, AJ, 106, 1547 (A93)

Aparicio, A., Bertelli, G., Chiosi, C., \& García-Pelayo, J. M. 1990, A\&A, 240,262

Aparicio, A., Bertelli, G., Chiosi, C., \& García-Pelayo, J. M. 1991, A\&AS, 88, 155

Arribas, S., \& Martínez-Roger, C. 1988, 206, 63

Boyle, R. J., \& McClure, R. D. 1975, PASP, 87, 12

Buser, R. 1978, A\&A, 62, 411

Buser, R., \& Kuruckz, R. L. 1978, A\&A, 70, 555

Cabrera-Caño, J., \& Alfaro, E. J. 1990, A\&A, 235, 94

Cameron, L. M. 1985, A\&A, 146, 59

Carney, B. W. 1979, ApJ, 233, 211

Carraro, G., Bertelli, G., Bressan, A., \& Chiosi, C. 1993, A\&AS, 101, 381

Castellani, V., Chieffi, A., \& Straniero, O. 1992, ApJS, 78, 517 (CAS)

Chiosi, C., Bertelli, G., \& Bressan, A. 1992, ARA\&A, 30, 235

Crawford, D. L., \& Mandwewala, N. 1976, PASP, 88, 917

Fagotto, F., Bressan, A., Bertelli, G., \& Chiosi, C. 1994, A\&AS, 104, 365
Flower, P. J. 1977, A\&A, 54, 31

Friel, E., \& Janes, K. A. 1993, A\&A, 267, 75

Geisler, D., Claria, J., \& Minniti, D. 1992, AJ, 104, 1892

Hartwick, F. D. A., \& Hesser, J. E. 1974, ApJ, 192, 391

Janes, K. A., \& Adler, D. 1982, ApJS, 49, 425

King, I. R. 1964, Royal Observatory Bulletin, No. 82, E-106

Landolt, A. U. 1983, AJ, 88, 439

Malagnini, M. L., Morossi, C., Rossi, L., \& Kuruckz, R. L. 1986, A\&A, 162,140

Meynet, G., Mermilliod, J. C., \& Maeder, A. 1993, A\&AS, 98, 477 (MEY)

Neckel, Th., \& Chini, R. 1980, A\&AS, 39, 41

Phelps, R. L., Janes, K. A., \& Montgomery, K. A. 1994, AJ, 107, 1079

Sandage, A. 1969, ApJ, 158, 115

Sandage, A., \& Eggen, O. J. 1959, MNRAS, 119, 278

Scalo, J. M. 1986, Fund. of Cosmic Phys., 11, 1

Stetson, P. B. 1987, PASP, 99, 191

Twarog, B. A., Anthony-Twarog, B. J., \& McClure, R. D., 1993, AJ (in press)

VandenBerg, D. A. 1983, ApJS, 51, 29

VandenBerg, D. A., \& Poll, H. E. 1989, AJ, 98, 1451 